\title{
Gastrointestinal defect closure using a novel through-the-scope helix tack and suture device compared to endoscopic clips in a survival porcine model (with video) $\square$
}

\section{(ㄷ)(i) $\Theta$}

\author{
Authors \\ Barham K. Abu Dayyeh', Navtej Buttar ${ }^{1}$, Andrew C. Storm ${ }^{1,2}$ \\ Institutions \\ 1 Division of Gastroenterology \& Hepatology, Mayo Clinic, \\ Rochester, Minnesota, United States \\ 2 Mayo Clinic Developmental Endoscopy Unit, Rochester, \\ Minnesota, United States \\ 3 Department of Comparative Medicine, Mayo Clinic, \\ Scottsdale, Arizona, United States
}

Ariosto Hernandez ${ }^{1,2}$, Neil B. Marya ${ }^{1}$, Tarek Sawas ${ }^{1}$, Elizabeth Rajan ${ }^{1,2}$, Naomi M. Gades ${ }^{3}$, Louis M. Wong Kee Song ${ }^{1}$,

submitted 16.9.2020

accepted after revision 23.11.2020

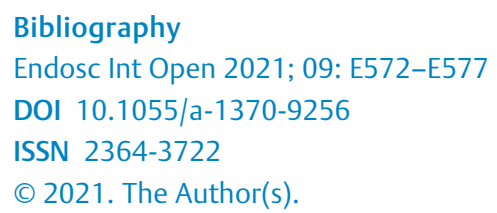
Commons Attribution-NonDerivative-NonCommercial License, permitting copying and reproduction so long as the original work is given appropriate credit. Contents may not be used for commercial purposes, or adapted, remixed, transformed or built upon. (https://creativecommons.org/licenses/by-nc-nd/4.0/)

Georg Thieme Verlag KG, Rüdigerstraße 14,

70469 Stuttgart, Germany

Corresponding author

Andrew C. Storm, MD, Mayo Clinic Developmental Endoscopy Unit, 200 First St SW, Rochester, MN 55905, United States

Fax: +1-507-255-7612

storm.andrew@mayo.edu

\section{ABSTRACT}

Background and aims Endoscopic resections are associated with bleeding and perforation and may be managed with through-the-scope (TTS) clips, over-the-scope clips and endoscopic suturing. The aim of this preclinical study was to compare technical success of closure using a novel TTS tissue helix tack and suture device (X-Tack) to TTS clips in a porcine model.

Materials and methods Four subjects underwent 40 mucosal resections, diameter range $25-50 \mathrm{~mm}$, in the stomach $(n=24)$ and colon $(n=16)$. Closures were randomized to $X$ Tack $(n=24)$ or clip $(n=16)$. Animals underwent weekly endoscopic follow-up for 4 weeks.

Results Technical closure with X-Tack was successful in 24 of $24(100 \%)$ cases and with clips in 13 of 16 cases $(81.3 \%)$ $(P=0.0001)$. One colonic perforation occurred and was successfully managed using X-Tack. The rate of healing was not statistically different between the groups, and all sites healed at 4 weeks including the perforation and were confirmed by histology.

Conclusions Compared to TTS clip, X-Tack is superior for effecting large mucosal defect closure, including durable sealing of full-thickness perforation. There was no difference in rate of healing between devices.

\section{Introduction}

Endoscopic resection of gastrointestinal neoplasia is increasingly being performed. Adverse events (AEs) related to endoscopic tissue resection include perforation and bleeding. Previous studies have reported decreased post-procedure bleeding and perforation with use of resection site closure [1-3].

Several devices are available to close such defects, each with associated limitations [4-7]. Over-the-scope clips (OTSC) and endoscopic suturing require endoscope removal for loading and then reinsertion to the target site, which may be impossi- ble or challenging. Therefore, a through-the-scope (TTS) tool capable of robust closure of large defects would be appealing. As such, a new device that overcomes the aforementioned limitations has been developed (X-Tack, Apollo Endosurgery, Austin, Texas, United States).

X-Tack is actuated through the 2.8-mm or larger channel of any gastroscope or colonoscope, without scope removal from the patient, and is designed as a series of helical coil tissue tacks, which are pre-strung on a suture string. Each helix tack is serially driven into tissue adjacent to the resection site, then drawn together, and finally cinched. The X-Tack is US Food and 
Drug Administration approved for clinical use as of December 2020.

The primary aim of this study was to compare immediate technical success with X-Tack relative to TTS clip placement in a survival porcine model of gastric and rectosigmoid mucosal defects. The secondary aim was to compare rates of mucosal defect healing over 4 weeks.

\section{Material and methods}

\section{Porcine Model}

Four adult Yorkshire/Large White crossbred pigs, mean weight $59.5 \mathrm{~kg}$ (range 56-65), three male castrate, were kept alive for 28 days after mucosal resection and closure procedures performed under general anesthesia. The animals were euthanized and necropsied after 28 days. The study was approved by the Mayo Clinic Institutional Animal Care and Use Committee.

\section{Devices}

The X-Tack device is a series of 5-mm-long surgical steel lasercut helical coil tacks (helix), preloaded on a 3-0 polypropylene suture strung through an eyelet on each tack, and deployed serially using a catheter placed through a protective liner within a $\geq 2.8$-mm endoscope working channel ( Fig. 1). Each helix is driven into tissue on the periphery of a mucosal defect using a Persian-drill driver handle rotating an inner catheter drive wire. The drill allows a fixed number of rotations, which limits the depth of penetration by the helix, generally to the level of the muscularis within the gastrointestinal tract wall. The eyelet also serves to prevent deeper penetration of the coil. Reversing the drill function will remove a helix from the tissue. After a helix is deployed, the driver catheter is removed from the endoscope and reloaded with the next helix over the same suture string. For this study, each suture was deployed using four helix tacks. After all tacks are placed, final tension is applied to the suture to achieve mucosal closure, followed by placement of a suture cinch (Apollo Endosurgery), identical to the cinch used with the OverStitch system, to fix the suture tension and close the resection site ( $\nabla$ Fig. 2 and $\triangleright$ Video $\mathbf{1}$ ).

For the comparative group, a commercially available TTS clip (Resolution 360, Boston Scientific, Natick, Massachusetts, United States) was used.

\section{Procedure}

On Day 0 , mucosal resection was performed using a hybrid technique involving installation of a submucosal fluid cushion (goal 3- to 4-cm diameter) followed by circumferential needle knife (Olympus America, Center Valley, Pennsylvania, United States) incision and en bloc snare (Lariat, Steris/US Endoscopy, Mentor, Ohio, United States) excision of the mucosa. Defects were measured using a guidewire with centimeter markings. A therapeutic gastroscope (GIF 2TH-180, Olympus America, Center Valley, Pennsylvania, United States) was used and all procedures were performed by a practicing therapeutic endoscopist.

In each animal, six mucosal resections were performed in the stomach. In the rectosigmoid colon, four mucosal defects were created. None of the lesions were left open or untreated.
In each animal, two gastric sites were randomized to closure with TTS clips and four with X-Tack. Two colon resection sites were randomized to closure with TTS clips and two with $X$ Tack. The number of devices needed to fully close a defect were unlimited, unless it was felt by the endoscopist after attempting the intended device that achieving closure was futile. In general, given the large size of the defects, a "zipper" technique was used for TTS clip closure [8]. If one modality failed to close the resection site adequately, the other device, or another tool if needed, was available but considered a technical failure of the initial device. Technical success was defined as mucosal closure of the defect with inability to visualize any significant portion of the resection bed.

Following defect closure, endoscopy and sigmoidoscopy were performed once weekly over a 28-day period to assess healing of the resection sites using a modified Forrest [9] visual analog score: Mayo Developmental Endoscopy Unit Score for Mucosal Resection Bed Healing:

IA: Transmural perforation

IB: Muscle injury evident

IIA: Active bleeding

IIB: Bleeding stigmata (visible vessel, adherent clot)

III: Clean based ulcer

IV: No mucosal defect (healed ulcer)

\section{Statistical analysis}

A descriptive analysis using means and frequencies for all variables was reported collectively and separately for stomach and colon. Data were analyzed in JMP version 14 (SAS Institute Inc., Cary, NC) using parametric and non-parametric tests with $P=<0.05$ as statistically significant. Comparisons of diameter of residual defect, weekly percentage of healing, closure time, and number of residual devices during follow-up were made. Subgroup analysis for lesions $>30 \mathrm{~mm}$ was performed. National Aeronautics and Space Administration (NASA) Task Load Index was performed to determine user workload of each device [10].

\section{Results}

In total, 40 mucosal defects were created (10 defects per animal), including 24 gastric and 16 colonic. Technical closure with X-Tack was successful in 24 of 24 (100\%). Technical closure with clips was successful in 13 of 16 (81.3\%). The first two clip failures involved gastric defects of $42 \mathrm{~mm}$ and $31 \mathrm{~mm}$ where seven of seven and four clips were attempted, respectively. Failure was attributed to size and shape of the defects. In both cases, successful salvage closure with X-Tack was performed ( $\triangleright$ Fig. 3 and $\triangleright$ Video 1 ). An additional TTS clip site required assistance apposing the edges using an alligator forceps through the second accessory channel.

Only one AE occurred during creation of a colonic defect, in which a 5-mm perforation occurred during supplemental snare excision to increase defect diameter. This was successfully closed with a single X-Tack system without clinical sequelae ( $\triangleright$ Fig. 3 and $\triangleright$ Video 1 ). There were no AEs attributed to closure devices. 


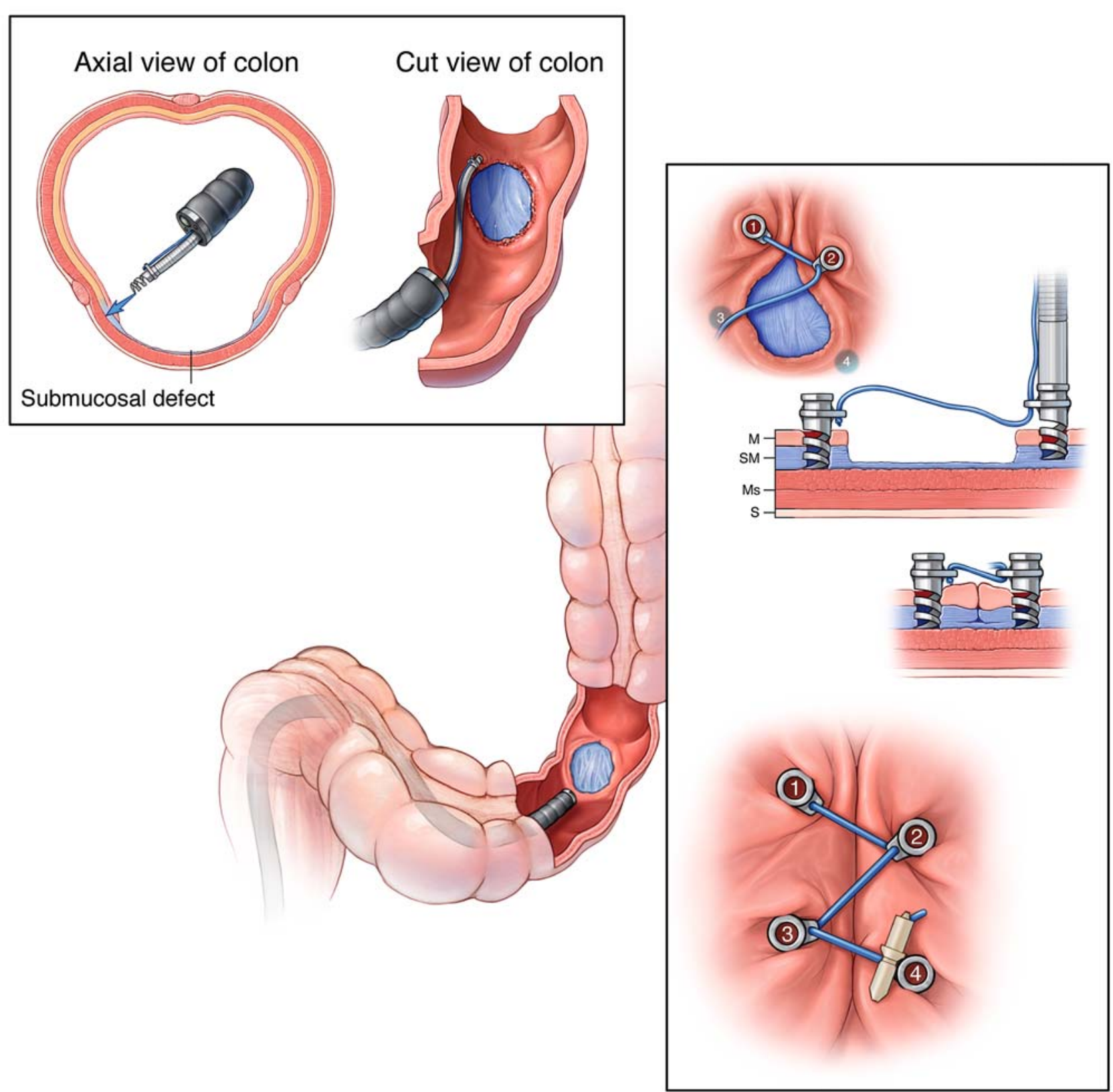

Fig. 1 X-Tack device. A series of surgical steel helix tacks strung on a 3-0 polypropylene suture are passed through the scope on a deployment catheter. Each tack is placed sequentially and then the coaxial suture is tightened and cinched to close a gastrointestinal tract defect. (Source: Mayo Foundation for Medical Education and Research)

The mean procedure time for $\mathrm{X}$-Tack was twice as long relative to TTS clips $(P=0.001)$. However, the resection diameter was larger in the $X$-Tack group compared to the clip group $(32.1 \pm 5.8 \mathrm{~mm}$ vs $28.3 \pm 5.2 \mathrm{~mm}$, respectively, $P=0.04)$ ( $\triangleright \mathrm{Ta}-$ ble 1).

NASA Task Load Index was calculated, with "performance" weighted the greatest for both devices (0.333) followed by "frustration" (0.267), "mental demand" (0.2), "temporal demand" (0.134), "effort" (0.067) and "physical demand" (0). Workload index (scaled from 0-100; 100 being the most possi- bly difficult task) was 23 for the X-Tack device, and 32.3 for TTS clips.

All resection sites achieved complete healing (stage IV) at Week 4 in both groups. The mean time to stage IV healing occurred at 2.5 weeks $(95 \% \mathrm{Cl} 2.2$ to 2.8$)$ for X-Tack and 2.3 weeks $(95 \% \mathrm{Cl} 1.7$ to 2.8$)$ for TTS clips $(P=0.55)$ ( $\mathbf{F i g} .4 a)$. For resections $>30 \mathrm{~mm}$, mean time to stage IV healing was 2.4 weeks $(95 \% \mathrm{Cl} 2.1$ to 2.8$)$ vs 2.7 weeks $(95 \% \mathrm{Cl} 1.9$ to 3.4$)$ for $\mathrm{X}$-tack and TTS clips, respectively $(P=0.51)$ ( $\triangleright$ Fig. $4 \mathbf{b})$. The X-tack device was retained at 4 weeks significantly more often than TTS clips ( $>$ Table $\mathbf{1}$ ). Histologic examination of all resection and 

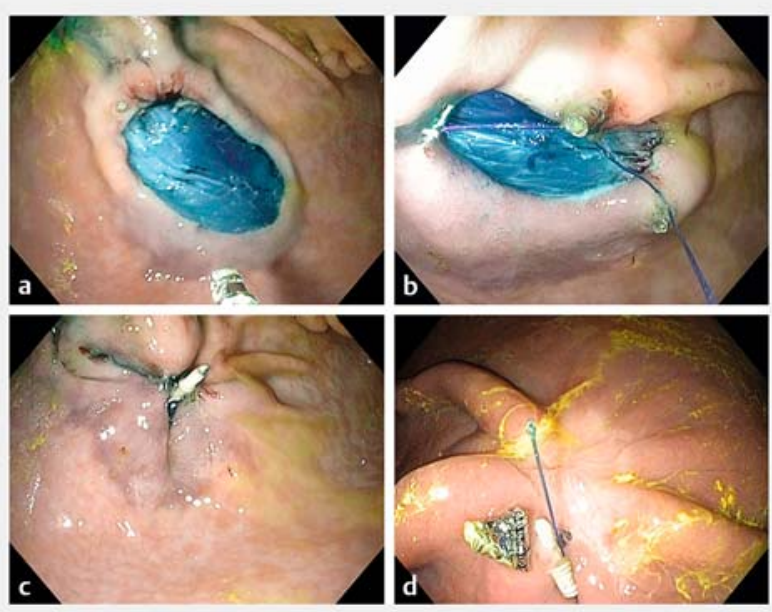

- Fig. 2 X-Tack procedure. a Open defect after mucosal resection. b Helix coil tacks placed and sequentially drilled into tissue on the periphery of the mucosal defect. c Defect closed after deploying a cinch. $\mathbf{d}$ Appearance of the healed ulcer with partially retained $\mathrm{X}$-Tack device on Day 21.

closure sites after necropsy confirmed healing, and mild inflammation was comparable between X-Tack and clip sites ( Fig.5).

\section{Discussion}

In this preclinical study, X-Tack resulted in significant improvement in immediate technical success, non-statistically different healing rates at 2 and 4 weeks, and a trend toward more rapid healing for lesions $>30 \mathrm{~mm}$ when compared to TTS clip closure. Furthermore, X-Tack was successful in closing two resection sites where TTS clips failed. One colonic defect with perforation was successfully managed using only the X-Tack device and healed without sequelae, a substantial advantage for perfora-

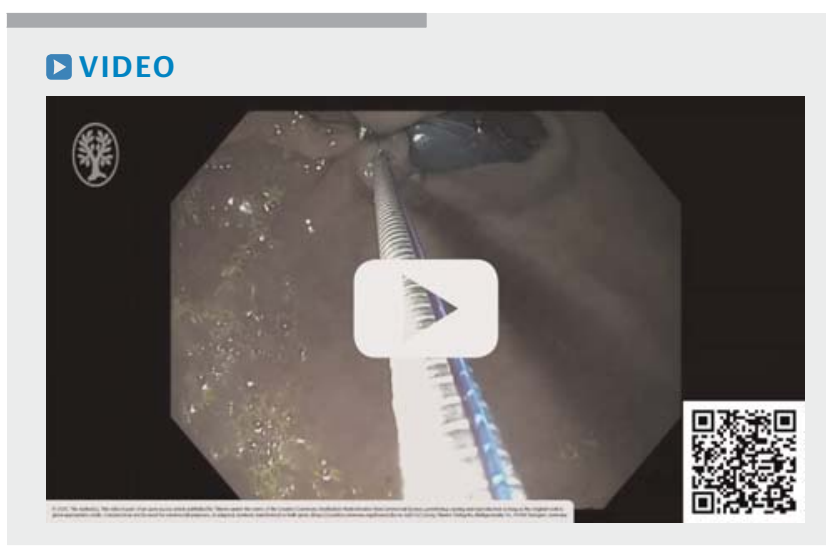

- Video 1 Video demonstrates: 1) routine closure of a gastric resection site using the novel helix tack and suture device; 2 ) helix tack and suture device closure of a gastric resection site that failed endoscopic clip closure; and 3) helix tack and suture device closure of a colonic perforation.

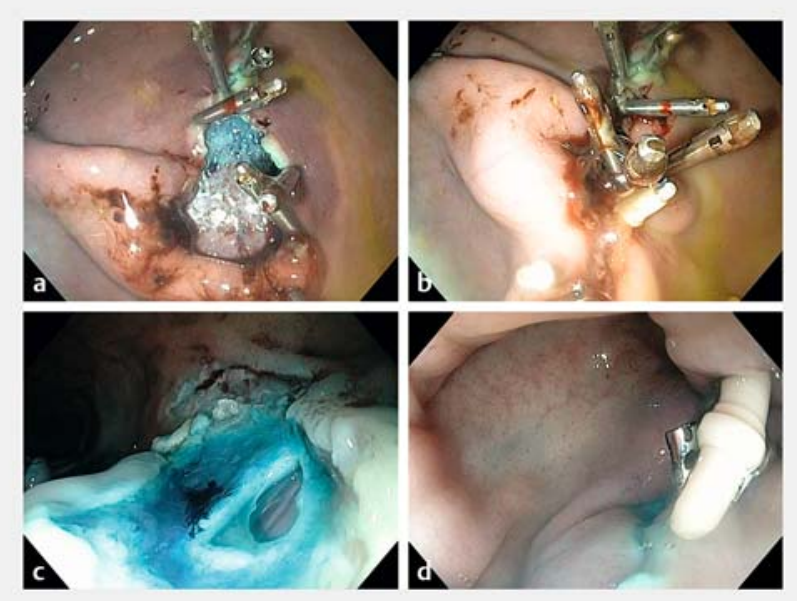

- Fig. 3 Rescue therapy with X-Tack following failed closure with TTS clips and X-Tack closure of a colonic perforation. a Failed closure attempt with TTS clips. b Successful completion of defect closure after with the X-Tack device. c Colonic mucosal resection complicated by a 5 -mm perforation. $\mathbf{d}$ Closure of the perforation site with X-tack.

tion closure in challenging colons or sites that are difficult to reaccess with bulkier devices like OTSC that require scope withdrawal for device loading and subsequent reinsertion.

Use of the X-tack device in the smaller-caliber porcine colon was simple and effective in all sites randomized to X-Tack closure; therefore, we anticipate that use in a human colon will also be feasible. Furthermore, because the X-Tack device can be used "on the fly" without needing to withdraw the scope from the patient for loading of the device like with endoscopic suturing and OTSC, we anticipate future use of the device will be of particular benefit for challenging right side colonic defects and perforations.

Increased procedural time for X-Tack closure may be explained by the learning curve, as well as more complicated and larger defect closures performed using X-Tack.

Despite increased closure times, NASA task load indices suggested that X-Tack was associated with overall lower user workload as compared to clips, driven by "performance" and "frustration" issues with closing large defects with TTS clips. Future studies should compare X-Tack to other robust closure devices, including over-the-scope clips and endoscopic suturing. As with these other closure devices, cost effectiveness of the $\mathrm{X}$ Tack device will be a consideration, and will require future study once the device is marketed and price-points become available.

Given higher rates of retention than TTS clips at 4 weeks, future use of X-Tack for endoscopic marking and anchoring of stents or feeding tubes is anticipated, and requires further study. Previous data suggest a mean retention interval of 1 to 2 weeks for TTS clips [11]. The X-Tack device, being small and made of surgical grade steel, is considered MRI conditional, similar to TTS clips. Long-term safety of the device for use as a marking device for example has not yet been studied. 
- Table 1 Comparison of X-Tack vs through-the-scope clip performance.

\begin{tabular}{|c|c|c|c|}
\hline Variable & $\begin{array}{l}X-T a c k(n=24) \\
\text { Mean } \pm S D / \%\end{array}$ & $\begin{array}{l}\text { Clips }(n=16) \\
\text { Mean } \pm S D / \%\end{array}$ & $P$ value \\
\hline \multicolumn{3}{|l|}{ Anatomic site } & \multirow[t]{3}{*}{0.29} \\
\hline - Stomach & $66.7 \%(n=16)$ & $50 \%(n=8)$ & \\
\hline - Colon & $33.3 \%(n=8)$ & $50 \%(n=8)$ & \\
\hline Successful closure & $100 \%(24 / 24)$ & $81.3 \%(13)$ & 0.13 \\
\hline Closure time (min) & $7.7 \pm 3.31$ & $3.9 \pm 3.3$ & 0.001 \\
\hline Induced lesion diameter (mm) & $32.1 \pm 5.8$ & $28.3 \pm 5.2$ & 0.04 \\
\hline Follow-up final diameter (mm) & $0.3 \pm 1.2$ & $0.4 \pm 1.0$ & 0.74 \\
\hline Number of devices deployed during initial closure & $1.0 \pm 0.2$ & $4.3 \pm 1.4$ & 0.000 \\
\hline Residual devices at necropsy & $0.8 \pm 0.4$ & $0.3 \pm 0.9$ & 0.043 \\
\hline
\end{tabular}

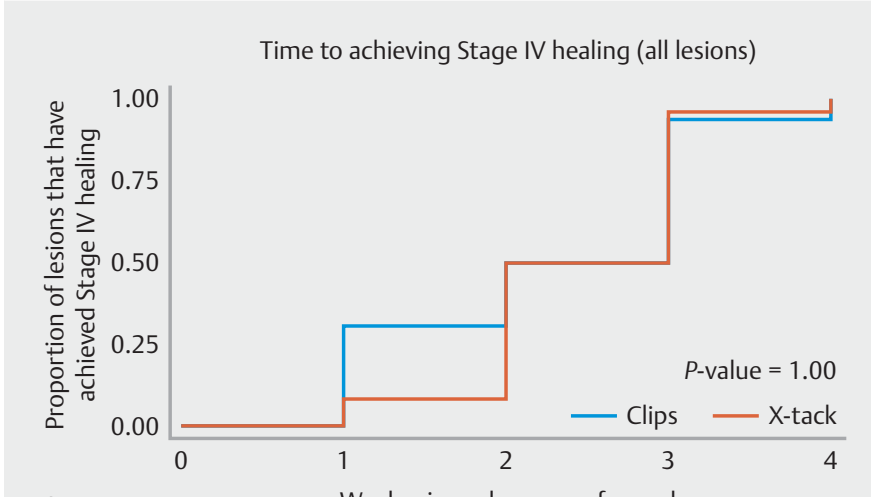

a

Weeks since closure performed
Time to achieving Stage IV healing (lesions greater than or equal to $30 \mathrm{~mm}$ )

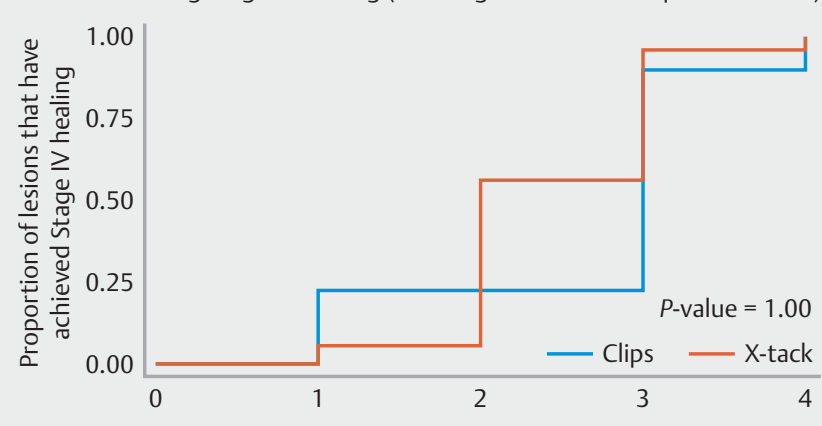

b

Weeks since closure performed

- Fig. 4 Kaplan-Meier curves showing a the proportion of lesions achieving stage IV (complete) ulcer healing on weekly examination between endoscopic clips and the X-Tack system and $\mathbf{b}$ the same for the largest lesions (>30 mm).

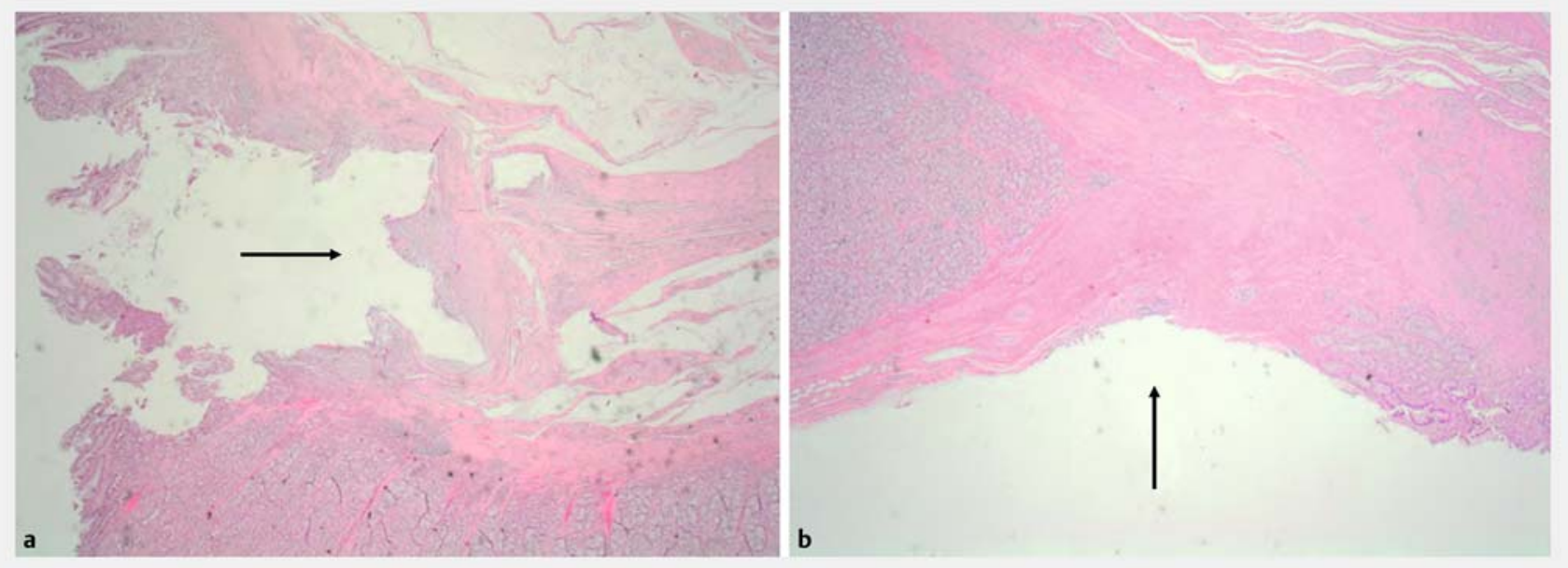

Fig. 5 Resection sites at 4 weeks showed similar healing at the defect closure and device implantation sites, with small residual areas devoid of mucosa (arrows) and mild inflammation with a mixed inflammatory cell infiltrate composed mostly of lymphocytes both in a X-Tack and b TTS clip closure sites (H\&E stain, $30 \times$ magnification). 


\section{Conclusions}

In conclusion, X-Tack is superior for acute closure of large resection defects compared to TTS clips in a porcine model, with similar rates of healing at 4 weeks. The perceived advantages of $X$-Tack over current large defect closure devices include relative simplicity and accuracy of use, without the need for endoscope withdrawal for device loading, and the ability to close larger lesions when TTS clips are inadequate.

\section{Competing interests}

This study was funded by a research grant from Apollo Endosurgery. Dr. Abu Dayyeh has received a research grant from Apollo Endosurgery. Dr. Storm has received a research grant and consulting fees from Apollo Endosurgery and consulting fees from ERBE.

\section{References}

[1] Raju GS. Closure of defects and management of complications. Gastrointest Endosc Clin N Am 2019; 29: 705-719

[2] Albeniz E, Alvarez MA, Espinos JC et al. Clip closure after resection of large colorectal lesions with substantial risk of bleeding. Gastroenterology 2019; 157: 1213-1221.e4
[3] Sakurai T, Adachi T, Kono M et al. Prophylactic suturing closure is recommended after endoscopic treatment of colorectal tumors in patients with antiplatelet/anticoagulant therapy. Oncology 2017; 93: 27-29

[4] Wang Z], Li SY, Zhang YH et al. Endoscopic closure of large colonic perforations with a novel endoscopic clip device: An animal study (with videos). J Gastroenterol Hepatol 2019; 34: 2152-2157

[5] Watson A, Zuchelli T. Repair of upper-Gl fistulas and anastomotic leakage by the use of endoluminal vacuum-assisted closure. VideoGIE 2019; 4: 40-44

[6] Al-Bawardy B, Rajan E, Wong Kee Song LM. Over-the-scope clip-assisted endoscopic full-thickness resection of epithelial and subepithelial Gl lesions. Gastrointest Endosc 2017; 85: 1087-1092

[7] Lopez-Nava G, Asokkumar R, Rull A et al. Safety and feasibility of a novel endoscopic suturing device (EndoZip TM) for treatment of obesity: first-in-human study. Obes Surg 2020; 30: 1696-1703

[8] Tang S]. Zipper clip closure of colonoscopic perforations. Gastrointest Endosc 2017; 85: 867-869

[9] Forrest JA, Finlayson ND, Shearman D]. Endoscopy in gastrointestinal bleeding. Lancet 1974; 2: 394-397

[10] Hart SG, Staveland LE. Development of NASA-TLX (Task Load Index): Results of empirical and theoretical research. Advance Psychol 1988; 52: $39-183$

[11] Shin EJ, Ko CW, Magno P et al. Comparative study of endoscopic clips: duration of attachment at the site of clip application. Gastrointest Endosc 2007; 66: 757-761

\section{CORRECTION}

Ariosto Hernandez, Neil B. Marya, Tarek Sawas et al. Gastrointestinal defect closure using a novel throughthe-scope helix tack and suture device compared to endoscopic clips in a survival porcine model (with video)

Endoscopy International Open 2021; 4: E572-E577. DOI: $10.1055 / a-1370-9256$

In the above-mentioned article $>$ Table 1 was corrected. This was corrected in the online version on 04.05.2022. 